\title{
Variabilidade genética em algumas criações comerciais brasileiras de escargots (Helix aspersa, Müller, 1774)
}

[Genetic variation at eight isoenzyme loci in subpopulations of the edible snail (Helix aspersa, Müller, 1774)]

\author{
B.F. Vasconcellos ${ }^{1}$, E.P.B. Contel $l^{2}$ \\ ${ }^{1}$ Departamento de Zootecnia - UCG-Goiás \\ Caixa Postal 86 \\ 74605-010 - Goiânia, GO \\ ${ }^{2}$ Faculdade de Medicina de Ribeirão Preto - USP - Ribeirão Preto, SP
}

\begin{abstract}
RESUMO
Descreveram-se os marcadores isoenzimáticos e estimou-se a variabilidade genética de 20 subpopulações brasileiras de escargots (Helix aspersa). O estudo dos oito locos foi feito por eletroforese em gel de amido, em amostras com 30 indivíduos cada, obtidas em criatórios dos estados de Santa Catarina, São Paulo e Rio de Janeiro (uma, duas e 17 amostras, respectivamente). Observou-se polimorfismo nos locos das enzimas LAP, 6-PGD, PEP 2, PEP 1 e MDH, com três alelos nos três primeiros locos e dois nos demais. Os locos da ME, da SOD e da PGI apresentaram-se monomórficos. As freqüências gênicas de sete amostras ajustaram-se ao modelo de Hardy-Weinberg $(\mathrm{P}<0,05)$, e as de outras seis amostras ajustaram-se ao modelo de Wright $(\mathrm{P}<0,05)$, indicando que elas estão submetidas a diferentes regimes reprodutivos. Os desvios da panmixia para toda a população $\left(\mathrm{F}_{\mathrm{IT}}\right)$ e dentro das subpopulações $\left(\mathrm{F}_{\mathrm{IS}}\right)$ não foram significativos $(\mathrm{P} \geq 0,05)$. $\mathrm{O}$ desvio entre as subpopulações $\left(\mathrm{F}_{\mathrm{ST}}=0,0485\right)$ foi significativo $(\mathrm{P}<0,05) \mathrm{e}$ apontou pequena diferenciação entre elas. As estimativas de diversidade total (Ht), entre subpopulações (Dst) e dentro das subpopulações (Hs), indicaram que a diversidade genética é reduzida e sua maior parte encontra-se dentro das subpopulações, sugerindo uma base genética estreita para essa população. As distâncias genéticas também foram pequenas, não permitindo a construção de um dendrograma.
\end{abstract}

Palavras-chave: escargot, isoenzima, variação genética, distância genética

\begin{abstract}
In order to assess genetic variability in subpopulations of Helix aspersa, eight isoenzyme loci in 30 individuals in each of 20 subpopulations, obtained from breeders in Santa Catarina (1), São Paulo(2) and Rio de Janeiro (17) states of Brazil, were examined. Polymorphic loci included LAP, 6-PGD, PEP 2, PEP 1 and MDH, with three alelles at each of the first three loci and two at each of the others. The ME, SOD and PGI loci were monomorphic. Gene frequencies in 7 of 20 subpopulations were consistent with the Hardy-Wienberg equilibrium $(P<0.05)$, and 6 were consistent with Wright model, indicating that these subpopulations did not meet requirements for genotypic equilibrium to be achieved. Despite the fact that some $F$ values were high, $F_{I S}$ and $F_{I T}$ were not significantly different from zero $(P \geq 0.05)$. Although small, the $F_{S T}$ value (0.0485) was significant, suggesting small differences among populations. Most of the low genetic variation at isoenzyme loci was observed within subpopulations rather than among subpopulations, suggesting a small genetic basis for these samples. Estimated genetic distances among pairs of subpopulations also were low.
\end{abstract}

Keywords: edible snail, escargot, isoenzymes, genetic variation, genetic distance

Recebido em 15 de junho de 2004

Aceito em 10 de março de 2005

E-mail: brenovasconcellos@bol.com.br 


\section{INTRODUÇÃO}

Originários da bacia do Mediterrâneo, os escargots da espécie Helix aspersa estão entre os mais consumidos em países europeus, mas, sendo o abate bem superior à produção comercial, há registros do seu desaparecimento em algumas áreas de ocorrência natural (Lazaridou-Dimitriadou et al., 1998).

A avaliação da variabilidade genética de uma espécie é fundamental quando se pretende melhorá-la geneticamente. Os marcadores moleculares são úteis para essa avaliação porque não sofrem efeitos ambientais e porque são mais polimórficos que os marcadores morfológicos, produzindo melhores estimativas dessa variabilidade (Ferreira e Gratapaglia, 1998).

As isoenzimas são diferentes formas moleculares de uma enzima catalisando, na célula, a mesma reação. Elas são encontradas na maior parte dos tecidos e apresentam um padrão de herança codominante, permitindo a exata identificação dos genótipos. Além disso, ao contrário do que ocorre com outros marcadores baseados na técnica da amplificação do DNA, o trabalho com isoenzimas dispensa conhecimento prévio sobre o genoma da espécie que se quer estudar. Estas características fazem delas marcadores ideais para a abordagem inicial na avaliação de parâmetros genéticos populacionais em espécies pouco estudadas (Robinson, 1998).

Diversos estudos com marcadores isoenzimáticos envolvendo a espécie $H$. aspersa foram realizados com populações naturais da Europa e da África (Crook, 1982; Madec, 1991 a e b; Guiller et al., 1994; Guiller et al., 1996; Arnaud et al., 1999). Entretanto, na literatura consultada, não foram encontrados trabalhos sobre a variabilidade genética de populações domesticadas e tampouco trabalhos com essa espécie no Brasil.

O objetivo do presente trabalho foi descrever alguns marcadores isoenzimáticos em escargots (Helix aspersa) e realizar, com o uso desses marcadores, uma estimativa da variabilidade genética de algumas subpopulações brasileiras.

\section{MATERIAL E MÉTODOS}

Analisaram-se 20 amostras com pelo menos 30 indivíduos cada uma, obtidas em diferentes criações comerciais do Rio de Janeiro (amostras 1 a 17), de São Paulo (amostras 18 e 19) e de Santa Catarina (amostra 20). Para a formação de cada amostra, foram retirados, aleatoriamente, no máximo cinco animais das caixas de criação, de forma a minimizar a coleta de animais aparentados.

Um fragmento do hepato-pâncreas de cada animal foi macerado e diluído em $0,5 \mathrm{ml}$ de tampão fosfato $(0,01 \mathrm{M}$; $\mathrm{pH} 6,5)$, e foi submetido à eletroforese em gel de amido a $11 \%$, segundo Harris e Hopkinson (1977).

Estudaram-se as seguintes enzimas: leucina aminopeptidase (LAP - Enzime Comission (EC) 3.4.11.1), peptidase 1 (PEP 1 - EC 3.4.13) e peptidase 2 (PEP 2 - EC 3.4.13), em tampão TCLB, pH 7,2; malato desidrogenase (MDH EC 1.1.1.37), enzima málica (ME - EC 1.1.1.40) e superóxido dismutase (SOD - E.C. 1.15.1.1) em tampão Tris-citrato, $\mathrm{pH}$ 7,4; fosfoglicoisomerase (PGI - EC 5.3.1.9) e 6fosfogluconato desidrogenase (6-PGD - EC 1.1.1.43), em tampão fosfato, $\mathrm{pH} 7,0$.

As freqüências gênicas e genotípicas para cada loco foram calculadas por meio da contagem de alelos e genótipos. A freqüência relativa dos heterozigotos, ou seja, a heterozigosidade observada $\left(\mathrm{H}_{\mathrm{o}}\right)$ foi calculada pela divisão do número absoluto de heterozigotos pelo número de indivíduos da amostra. A heterozigosidade esperada $\left(\mathrm{H}_{\mathrm{e}}\right)$ foi calculada a partir do modelo de equilíbrio de Hardy-Weinberg, sendo: $\mathrm{He}=2 \Sigma\left[\mathrm{p}_{\mathrm{i}}\left(1-\mathrm{p}_{\mathrm{i}}\right)\right]$, em que:

$\mathrm{p}_{\mathrm{i}}=$ freqüência relativa do alelo $\mathrm{i}$ em cada amostra.

As comparações entre as freqüências genotípicas observadas e aquelas preditas pelo modelo de equilíbrio de Hardy-Weinberg foram feitas pelo teste exato de Haldane (1954), para os locos com apenas dois alelos, e pelo método de reamostragem (método de Monte Carlo), proposto por Guo e Thompson (1992). 
Para as amostras em que pelo menos um dos seus locos não se ajustou ao modelo de equilíbrio de Hardy-Weinberg, realizou-se o teste de $\chi^{2}$ para a verificação da adequação das suas freqüências genotípicas ao modelo de equilíbrio de Wright. Para tanto, foi estimado o índice médio de fixação ( $\overline{\mathrm{f}}$ ) de acordo com a equação:

$\overline{\mathrm{f}}=1-\left(\Sigma \mathrm{H}_{\mathrm{o}} / \Sigma \mathrm{H}_{\mathrm{e}}\right)$, em que:

$\Sigma \mathrm{H}_{\mathrm{o}}$ e $\Sigma \mathrm{H}_{\mathrm{e}}$ são iguais aos somátórios das heterozigosidades observadas e esperadas, respectivamente, para todos os locos polimórficos da amostra em questão.

Nos casos em que o $\overline{\mathrm{f}}$ foi diferente de zero, foram calculadas as freqüências genotípicas esperadas, segundo as equações do modelo de Wright:

$\mathrm{P}_{\mathrm{ii}}=\mathrm{p}_{\mathrm{i}}^{2}+\overline{\mathrm{f}} \mathrm{p}_{\mathrm{i}}\left(1-\mathrm{p}_{\mathrm{i}}\right) ;$

$\mathrm{P}_{\mathrm{ij}}=2 \mathrm{p}_{\mathrm{i}} \mathrm{p}_{\mathrm{j}}(1-\overline{\mathrm{f}}) ; \mathrm{e}$

$P_{j j}=p_{j}^{2}+\bar{f}_{j}\left(1-p_{j}\right)$, em que:

$p_{i}$ e $p_{j}$ representam as estimativas das freqüências alélicas dos alelos $\mathrm{i}$ e $\mathrm{j}$ e $\mathrm{P}_{\mathrm{ii}}, \mathrm{P}_{\mathrm{jj}}$ e $\mathrm{P}_{\mathrm{ij}}$ as freqüências esperadas de cada um dos genótipos, respectivamente. Esses cálculos foram realizados com o uso do programa Microsoft Excel.

Em relação aos locos que se apresentaram polimórficos, foram calculados, segundo Alfenas et al. (1991), as seguintes medidas: número médio de alelos por loco polimórfico $(\mathrm{A}), \mathrm{A}=$ número total de alelos dos locos polimórficos/número de locos polimórficos; número efetivo de alelos por loco $\left(\mathrm{A}_{\mathrm{e}}\right), \mathrm{A}_{\mathrm{e}}=1 / 1$ $\mathrm{H}_{\mathrm{e}}$, em que $\mathrm{H}_{\mathrm{e}}$ é igual à heterozigosidade esperada para o loco, segundo a equação de equilíbrio de Hardy-Weinberg; proporção de alelos presentes em cada $\operatorname{amostra}\left(\mathrm{P}_{\mathrm{a}}\right), \mathrm{P}_{\mathrm{a}}=$ número de alelos na amostra/número total de alelos; e proporção de locos polimórficos $(\mathrm{P})$, em que $\mathrm{P}=$ número de locos polimórficos na amostra/número de locos analisados.

Para a análise da diversidade e da estrutura genética das amostras estudadas, foram ainda calculados os índices de diversidade genética de Nei (1972) e as estatísticas F de Wright (Weir e Cockerham, 1984).

Os índices de diversidade genética de Nei (1973) foram calculados da seguinte forma:

$\left(\mathrm{H}_{\mathrm{T}}\right), \mathrm{H}_{\mathrm{T}}=1-\Sigma \overline{\mathrm{p}}_{\mathrm{i}}^{2}$, em que:
$\mathrm{H}_{\mathrm{T}}$ é a diversidade total e $\Sigma \overline{\mathrm{p}}_{\mathrm{i}}^{2}$ é o somatório das freqüências médias do alelo $i$ em um determinado loco em relação a todas as amostras; $\mathrm{H}_{\mathrm{S}}=\Sigma \mathrm{H}_{\mathrm{e}} / \mathrm{n}$, em que:

$\mathrm{H}_{\mathrm{S}}$ é a diversidade dentro da amostra e $\mathrm{H}_{\mathrm{e}}$ é a heterozigosidade esperada para cada loco em cada amostra e n é o número de amostras;

$\mathrm{D}_{\mathrm{ST}}=\mathrm{H}_{\mathrm{T}}-\mathrm{H}_{\mathrm{S}}$, em que:

$\mathrm{D}_{\mathrm{ST}}$ é a diversidade entre as amostras;

e $\mathrm{G}_{\mathrm{ST}}=\mathrm{D}_{\mathrm{ST}} / \mathrm{H}_{\mathrm{T}}$, em que:

$\mathrm{G}_{\mathrm{ST}}$ é a proporção da diversidade total atribuível à subdivisão da população.

As estatísticas $\mathrm{F}$ de Wright $\left(\mathrm{F}_{\mathrm{IT}}, \mathrm{F}_{\mathrm{ST}}\right.$ e $\left.\mathrm{F}_{\mathrm{IS}}\right)$, que estimam o grau de fixação gênica resultante da endogamia biparental, foram obtidas com as seguintes fórmulas:

$\mathrm{F}_{\mathrm{IT}}=1-\left(\overline{\mathrm{H}}_{\mathrm{o}} / \overline{\mathrm{H}}_{\mathrm{e}}\right)$, em que:

$\mathrm{F}_{\text {IT }}$ é o desvio de panmixia para a população e $\overline{\mathrm{H}}_{\mathrm{o}}$ e $\overline{\mathrm{H}}_{\mathrm{e}}$ são, respectivamente, as estimativas de heterozigosidade média observada e esperada para todas as amostras;

$\mathrm{F}_{\mathrm{ST}}=\sigma^{2}{ }_{\text {subpopulações }} / \mathrm{p}(1-\mathrm{p})$, em que:

$\mathrm{F}_{\mathrm{ST}}$ é o desvio de panmixia decorrente da subdivisão da população e $\mathrm{p}(1-\mathrm{p})$ equivale à variância da distribuição binomial das freqüências alélicas para todas as subpopulações; e $\mathrm{F}_{\mathrm{IT}}=1-\left(\mathrm{H}_{\mathrm{o}} / \mathrm{H}_{\mathrm{e}}\right)$, em que:

$\mathrm{F}_{\text {IT }}$ é o desvio de panmixia de cada população e $\mathrm{H}_{\mathrm{o}} \quad$ e $\mathrm{H}_{\mathrm{e}}$ são, respectivamente, as heterozigosidades observadas e esperadas para cada uma das amostras.

Para a realização de uma análise de agrupamento, foram calculadas, a partir das freqüências alélicas observadas em cada amostra, as estimativas das identidades (I) e das distâncias genéticas (D) entre cada par de amostras segundo Nei (1972).

O grau de identidade genética (I), que corresponde à probabilidade de que dois alelos tomados ao acaso, tanto entre como dentro de uma amostra sejam idênticos, foi dado por: $\mathrm{I}=\frac{\mathrm{J}_{\mathrm{pq}}}{\sqrt{\mathrm{J}_{\mathrm{p}}} \times \mathrm{J}_{\mathrm{q}}}, \quad$ em que: $\mathrm{J}_{\mathrm{pq}}=\Sigma \mathrm{p}_{\mathrm{i}} \mathrm{q}_{\mathrm{i}}$ é a probabilidade que dois alelos tomados ao acaso em duas amostras P e Q sejam idênticos, sendo $p_{i}$ e $\mathrm{q}_{\mathrm{i}}$ as freqüências do alelo $\mathrm{i}$ em cada uma das amostras; $\mathrm{J}_{\mathrm{p}}=\Sigma \mathrm{p}_{\mathrm{i}}^{2}$ e $\mathrm{J}_{\mathrm{q}}=\Sigma \mathrm{q}_{\mathrm{i}}^{2}$ são, respectivamente, as probabilidades de que dois 
alelos tomados ao acaso dentro de uma amostra (P ou Q) sejam idênticos.

O grau de distância genética (D), que corresponde ao cologaritmo neperiano de I, é igual a: $\mathrm{D}=-\ln (\mathrm{I})$

Com exceção do cálculo das freqüências genotípicas para a realização do teste de aderência ao modelo de Wright, todas as estimativas foram feitas com o auxílio do programa TFPGA (Miller, 1997).

\section{RESULTADOS E DISCUSSÃO}

Dos oito locos estudados, três apresentaram-se monomórficos (ME, SOD e PGI). Nos demais, foram observados dois (PEP 1 e $\mathrm{MDH}$ ) e três alelos (LAP, PEP 2 e 6-PGD), respectivamente. $\mathrm{O}$ padrão das bandas nos zimogramas das enzimas que se apresentaram polimórficas sugeriu uma estrutura monomérica para elas.

De modo geral, os resultados foram semelhantes aos encontrados na literatura consultada em relação à existência de polimorfismo em $H$. aspersa, nos locos correspondentes à LAP (Crook, 1982; Guiller et al., 1994; Arnaud et al., 1999), à PEP (Madec, 1991 a), à 6-PGD (Guiller, et al., 1994), à MDH (Madec, 1991 a; Guilleret al., 1994; Arnaud et al., 1999) e à ME (Guiller et al., 1994). Assemelham-se também aos resultados observados por Arnaud et al. (1999) com relação ao aspecto monomórfico da SOD.

O fato de os números de alelos por loco observados no presente trabalho serem bem inferiores a quase todos aqueles relatados na literatura consultada sugere menor variabilidade genética nessas amostras. A freqüência dos alelos de cada loco, em relação a todas as amostras e para cada uma individualmente, é apresentada na Tab. 1.

Com exceção do alelo 3 do primeiro loco (LAP) e do alelo 2 do último (MDH), nenhum dos alelos apresentou freqüência inferior a $1 \%$, considerando cada amostra isoladamente. No conjunto, todos os alelos apresentaram freqüências superiores a $1 \%$. Os alelos com freqüências inferiores a $5 \%$ foram observados nas amostras $2,4,5,6,7,8,9,11,13,14,15,16$, $17,18,19$ e 20 (LAP); 5, 11, 12, 13 (PEP2); 5 (6-PGD) e 3, 4, 5, 6, 8, 9, 10, 11, 12, 14, 16 e 17 $(\mathrm{MDH})$. Nas amostras 10 e 12 houve inclusive fixação do alelo 1 da $\mathrm{MDH}$.

As heterozigosidades observadas e esperadas, os índices médios de fixação ( $\bar{f}$ ) e as probabilidades exatas dos testes de aderência das freqüências genotípicas observadas aos modelos de equilíbrio de Hardy -Weinberg e de Wright, para cada uma das amostras, são apresentados na Tab. 2.

Das 20 amostras analisadas, em sete a probabilidade de ajuste ao modelo de equilíbrio de Hardy-Weinberg foi igual ou superior a $95 \%$ (amostras 3, 6, 7, 8, 14, 15 e 20). Das 13 amostras que não se ajustaram a esse modelo, seis ajustaram-se ao modelo de equilíbrio de Wright. Estes resultados indicam que as subpopulações de onde essas amostras foram obtidas estão submetidas a diferentes regimes reprodutivos e que nem todas são panmíticas com relação aos locos analisados.

Os $\overline{\mathrm{f}}$ positivos observados nas demais amostras indicaram menor freqüência de heterozigotos nas subpopulações do que aquela esperada pelo teorema de Hardy-Weinberg. Isso provavelmente é causado por endogamia não intencional, uma vez que todos os produtores consultados demonstraram preocupação em não acasalar animais cujo parentesco fosse muito próximo.

Índices médios de fixação positivos podem ser causados tanto por endogamia como por deriva genética. A hipótese de deriva é, contudo, pouco provável, uma vez que todos os heliários estudados contavam com, no mínimo, 500 reprodutores.

Contudo, a identificação das famílias nessas criações foi precária e, como os animais destinados à reprodução são mantidos em grupos, pode estar ocorrendo acasalamento entre parentes com maior freqüência do que entre animais não aparentados. Além disso, é comum os produtores utilizarem os animais adultos de sua própria criação para a reprodução, o que reforça a hipótese de haver endogamia. 


\section{Vasconcellos e Contel}

Tabela 1. Freqüências alélicas dos diferentes locos em cada amostra e no conjunto de todas as amostras

\begin{tabular}{|c|c|c|c|c|c|c|c|c|c|c|c|c|c|}
\hline & & Lap & & $\mathrm{Pe}$ & $\mathrm{p} 1$ & & Pep2 & & & 6-Pgd & & M & dh \\
\hline & 1 & 2 & 3 & 1 & 2 & 1 & 2 & 3 & 1 & 2 & 3 & 1 & 2 \\
\hline 1 & 0,7759 & 0,2069 & 0,1720 & 0,7500 & 0,2500 & 0,3103 & 0,4310 & 0,2586 & 0,3833 & 0,4833 & 0,1333 & 0,9310 & 0,0690 \\
\hline 2 & 0,6833 & 0,3000 & 0,0167 & 0,6833 & 0,3167 & 0,5517 & 0,3276 & 0,1207 & 0,1724 & 0,5345 & 0,2931 & 0,9483 & 0,0517 \\
\hline 3 & 0,8500 & 150 & 0,0000 & 0,6724 & 0,3276 & 0,4464 & 0,3393 & 0,2143 & 0,2069 & 0,5172 & 0,2759 & 0,9828 & 0,0172 \\
\hline 4 & 0,6552 & 0,3276 & 0.0172 & 0.5333 & 0.4667 & 0.4000 & 0.5333 & 0.0667 & 0.4500 & 0.4500 & 0.1000 & 0.9667 & 0.0333 \\
\hline 5 & 0.4167 & 0.5833 & 0.0000 & 0.6000 & 0.4000 & 0.2759 & 0.7069 & 0.0172 & 0.6500 & 0.3167 & 0.0333 & 0.9833 & 0.0167 \\
\hline 6 & 0.7167 & 0.2833 & 0.0000 & 0.6290 & 0.3710 & 0.5357 & 0.4107 & 0.0536 & 0.5172 & 0.3966 & 0.0862 & 0.9655 & 0.0345 \\
\hline 7 & 0.7500 & 0.2500 & 0.0000 & 0.6207 & 0.3793 & 0.2333 & 0.6167 & 0.1500 & 0.5000 & 0.4333 & 0.0667 & 0.9310 & 0.0690 \\
\hline 8 & 0.7000 & 0.3000 & 0.0000 & 0.8500 & 0.1500 & 0.2500 & 0.4333 & 0.3167 & 0.3833 & 0.4333 & 0.1833 & 0.9833 & 0.0167 \\
\hline 9 & 0.3621 & 0.6207 & 0.0172 & 0.6167 & 0.3833 & 0.1833 & 0.6500 & 0.1667 & 0.4333 & 0.4333 & 0.1333 & 0.9667 & 0.0333 \\
\hline 10 & 0.3500 & 0.6000 & 0.0500 & 0.5833 & 0.4167 & 0.4000 & 0.5500 & 0.0500 & 0.4167 & 0.3833 & 0.2000 & 0.1000 & 0.0000 \\
\hline 11 & 0.2857 & 0.6964 & 0.0179 & 0.6296 & 0.3704 & 0.2586 & 0.7241 & 0.0172 & 0.3833 & 0.4500 & 0.1667 & 0.9500 & 0.0500 \\
\hline 12 & 0.2833 & 0.6667 & 0.0500 & 0.4655 & 0.5345 & 0.1897 & 0.7931 & 0.0172 & 0.4483 & 0.4138 & 0.1379 & 1.0000 & 0.0000 \\
\hline 13 & 0.7241 & 0.2414 & 0.0345 & 0.6607 & 0.3393 & 0.3103 & 0.5172 & 0.1724 & 0.4167 & 0.4500 & 0.1333 & 0.9167 & 0.0833 \\
\hline 14 & 0.8214 & 0.1786 & 0.0000 & 0.6296 & 0.3704 & 0.0385 & 0.5577 & 0.4038 & 0.2414 & 0.5172 & 0.2414 & 0.9655 & 0.0345 \\
\hline 15 & 0. & 0. & 0. & 0 . & 0 & 00 & 0 & 0.1 & 0.2 & 67 & 0.3 & 0.9167 & 0.0833 \\
\hline 16 & 0.5172 & 0.4828 & 0.0000 & 0.5517 & 0.4483 & 0.2069 & 0.6207 & 0.1724 & 0.3793 & 0.4483 & 0.1724 & 0.9643 & 0.0357 \\
\hline 17 & 0.7586 & 0.2414 & 0.0000 & 0.6071 & 0.3929 & 0.3667 & 0.4333 & 0.2000 & 0.4333 & 0.4000 & 0.1667 & 0.9667 & 0.0333 \\
\hline 18 & 0.6552 & 0.3276 & 0.0172 & 0.7833 & 0.2167 & 0.3667 & 0.4500 & 0.1833 & 0.4833 & 0.3833 & 0.1333 & 0.9500 & 0.0500 \\
\hline 19 & 0.5862 & 0.4138 & 0.0000 & 0.6167 & 0.3833 & 0.2167 & 0.6333 & 0.1500 & 0.4500 & 0.3833 & 0.1667 & 0.9500 & 0.0500 \\
\hline 20 & 0.5517 & 0.4310 & 0.0172 & 0.8889 & 0.1111 & 0.2586 & 0.4655 & 0.2759 & 0.3167 & 0.5333 & 0.1500 & 0.8448 & 0.1552 \\
\hline Total & 0,6067 & 0,3797 & 0,0137 & 0,6538 & 0,3462 & 0,3134 & 0,5308 & 0,1558 & 0,3948 & 0,4411 & 0,1641 & 0,9542 & 0,0458 \\
\hline
\end{tabular}

Lap = leucina aminopeptidase; Pep 1 = peptidase $1 ;$ Pep2 = peptidase 2; 6-Pgd = 6-fosfogluconato desidrogenase; Mdh = malato desidrogenase.

Eventuais efeitos negativos da endogamia nas criações podem não estar sendo percebidos pelos produtores justamente pela falta de registros genealógicos, especialmente se se considerar que algumas amostras $(4,9,10$ e 12) apresentaram índices médios de fixação superiores a $20 \%$. É importante observar que a análise de uma única geração é insuficiente para que se descreva, de forma segura, o regime reprodutivo a que cada subpopulação está submetida. Além disso, no presente estudo não foi possível afirmar que certas condições para a aplicação dos modelos de equilíbrio, tais como neutralidade seletiva dos alelos e independência de segregação entre os locos estudados, tenham sido obedecidas.

O valor observado para o desvio médio de panmixia (Tab. 3) em relação a todas as subpopulações $\left(\mathrm{F}_{\mathrm{IT}}\right)$ não foi significativo, indicando que o conjunto das subpopulações não apresentou falta ou excesso de heterozigotos. 
Tabela 2. Heterozigosidade observada (Ho) e esperada (He) para cada loco em cada amostra, probabilidade do teste exato de Haldane (P) ou do teste por meio do algoritmo de Monte Carlo (MC) para verificação do equilíbrio de Hardy-Weinberg em relação a cada loco, índice médio de fixação de cada amostra $(\overline{\mathrm{f}})$ e probabilidade do teste de $\chi^{2}$ para a verificação do equilíbrio com endogamia de Wright

\begin{tabular}{|c|c|c|c|c|c|c|c|c|c|c|c|c|c|c|c|c|c|}
\hline & & Lap & & & Pep1 & & & Pep2 & & & $6-\operatorname{Pgd}$ & & & Mdh & & $\bar{f}$ & $\gamma^{2}$ \\
\hline & $\mathrm{He}$ & Но & $\mathrm{MC}$ & $\mathrm{He}$ & Ho & $\mathrm{P}$ & $\mathrm{He}$ & Ho & $\mathrm{MC}$ & $\mathrm{He}$ & Ho & $\mathrm{MC}$ & $\mathrm{He}$ & Ho & $\mathrm{P}$ & 1 & $\chi$ \\
\hline 1 & 0,3549 & 0,3793 & 1,0000 & 0,3750 & 0,1667 & 0,0047 & 0,6510 & 0,5172 & 0,4176 & 0,6017 & 0,5667 & 0,7322 & 0,1284 & 0,1379 & 1,00 & 0,1625 & $8,95^{\mathrm{NS}}$ \\
\hline 2 & 0,4428 & 0,3000 & 0,0763 & 0,4328 & 0,2333 & 0,0140 & 0,5737 & 0,5172 & 0,1095 & 0,5987 & 0,6897 & 0,2859 & 0,0981 & 0,1034 & 1,00 & 0,1409 & $15,40^{\mathrm{NS}}$ \\
\hline 3 & 0,2550 & 0,3000 & 1,0000 & 0,4405 & 0,2414 & 0,2830 & 0,6397 & 0,5357 & 0,1367 & 0,6136 & 0,7241 & 0,2328 & 0,0339 & 0,0345 & 1,00 & 0,0741 & -- \\
\hline 4 & 0,4631 & 0,2414 & 0,0048 & 0,4978 & 0,3333 & 0,0749 & 0,5511 & 0,4333 & 0,3025 & 0,5850 & 0,4667 & 0,0970 & 0,0644 & 0,0667 & 1,00 & 0,2868 & $15,17^{\mathrm{NS}}$ \\
\hline 5 & 0,4861 & 0,1667 & 0,0002 & 0,4800 & 0,6000 & 0,2643 & 0,4239 & 0,3103 & 0,2125 & 0,4761 & 0,5333 & 0,7093 & 0,0328 & 0,0333 & 1,00 & 0,1344 & $11,59^{\mathrm{NS}}$ \\
\hline 6 & 0,4061 & 0,3667 & 0,6602 & 0,4667 & 0,4839 & 1,0000 & 0,5415 & 0,5357 & 0,2108 & 0,5678 & 0,6897 & 0,1361 & 0,0666 & 0,0690 & 1,00 & $-0,0470$ & -- \\
\hline 7 & 0,3750 & 0,4333 & 0,6379 & 0,4709 & 0,5517 & 0,4575 & 0,5428 & 0,6000 & 0,5750 & 0,5578 & 0,4667 & 0,0695 & 0,1284 & 0,1379 & 1,00 & $-0,0552$ & -- \\
\hline 8 & 0,4200 & 0,3333 & 0,3807 & 0,2550 & 0,1667 & 0,0991 & 0,6494 & 0,6000 & 0,1399 & 0,6317 & 0,7667 & 0,2020 & 0,0328 & 0,0333 & 1,00 & 0,0446 & -- \\
\hline 9 & 0,4834 & 0,2759 & 0,0140 & 0,4728 & 0,3000 & 0,0546 & 0,5161 & 0,4333 & 0,1322 & 0,6067 & 0,5333 & 0,0048 & 0,0644 & 0,0667 & 1,00 & 0,2492 & $23,25 * *$ \\
\hline 10 & 0,5150 & 0,2667 & 0,0024 & 0,4861 & 0,3000 & 0,0563 & 0,5350 & 0,5667 & 0,7636 & 0,6394 & 0,6000 & 0,0374 & 0,0000 & 0,0000 & -- & 0,2032 & $25,38^{* *}$ \\
\hline 11 & 0,4330 & 0,3571 & 0,1504 & 0,4664 & 0,3704 & 0,4051 & 0,4084 & 0,2414 & 0,0136 & 0,6228 & 0,6000 & 0,3878 & 0,0950 & 0,1000 & 1,00 & 0,1760 & $15,70^{\mathrm{NS}}$ \\
\hline 12 & 0,4728 & 0,2000 & 0,0001 & 0,4976 & 0,3793 & 0,2618 & 0,3347 & 0,1724 & 0,0050 & 0,6880 & 0,5172 & 0,0329 & 0,0000 & 0,0000 & -- & 0,3633 & $31,54 * *$ \\
\hline 13 & 0,4162 & 0,4138 & 1,0000 & 0,4483 & 0,3214 & 0,1995 & 0,6064 & 0,6897 & 0,8745 & 0,6061 & 0,7000 & 0,0111 & 0,1528 & 0,1667 & 1,00 & $-0,0277$ & $45,61^{* *}$ \\
\hline 14 & 0,2934 & 0,2857 & 1,0000 & 0,4664 & 0,4444 & 1,0000 & 0,5244 & 0,6154 & 0,7210 & 0,6159 & 0,7241 & 0,2715 & 0,0666 & 0,0690 & 1,00 & $-0,0874$ & -- \\
\hline 15 & 0,4382 & 0,4138 & 0,7627 & 0,4082 & 0,2857 & 0,1568 & 0,6061 & 0,6000 & 0,0967 & 0,6350 & 0,7000 & 0,5459 & 0,1528 & 0,1667 & 1,00 & 0,0330 & -- \\
\hline 16 & 0,4994 & 0,2759 & 0,0235 & 0,4946 & 0,5517 & 0,7129 & 0,5422 & 0,4138 & 0,0590 & 0,6254 & 0,7241 & 0,0422 & 0,0689 & 0,0714 & 1,00 & 0,0867 & $22,69 * *$ \\
\hline 17 & 0,3662 & 0,3727 & 1,0000 & 0,4770 & 0,3571 & 0,2342 & 0,6378 & 0,6333 & 0,1262 & 0,6244 & 0,6000 & 0,3945 & 0,0644 & 0,0667 & 1,00 & 0,0645 & $18,84^{*}$ \\
\hline 18 & 0,4631 & 0,2759 & 0,0281 & 0,3394 & 0,3667 & 1,0000 & 0,6294 & 0,5667 & 0,4430 & 0,6017 & 0,6000 & 0,1326 & 0,0950 & 0,1000 & 1,00 & 0,1030 & $13,49^{\mathrm{NS}}$ \\
\hline 19 & 0,4851 & 0,4138 & 0,4541 & 0,4728 & 0,3000 & 0,0546 & 0,5294 & 0,4000 & 0,1676 & 0,6228 & 0,7333 & 0,0045 & 0,0950 & 0,1000 & 1,00 & 0,1170 & $26,29 * *$ \\
\hline 20 & 0,5095 & 0,5517 & 1,0000 & 0,1975 & 0,1481 & 0,2665 & 0,6403 & 0,6207 & 0,8675 & 0,5928 & 0,6000 & 0,8625 & 0,2622 & 0,3103 & 1,00 & $-0,0129$ & -- \\
\hline
\end{tabular}


Tabela 3. Estimativas médias para os índices de fixação de Wright obtidas a partir de marcadores isoenzimáticos

\begin{tabular}{lccc}
\hline Loco & $\mathrm{F}_{\mathrm{IT}}$ & $\mathrm{F}_{\mathrm{ST}}$ & $\mathrm{F}_{\mathrm{IS}}$ \\
\hline LAP & 0,3219 & 0,1067 & 0,2408 \\
PEP 1 & 0,2389 & 0,0258 & 0,2187 \\
PEP 2 & 0,1638 & 0,0545 & 0,1156 \\
6- PGD & $-0,0040$ & 0,0193 & $-0,0238$ \\
MDH & $-0,0465$ & 0,0123 & $-0,0595$ \\
Média & $0,1562 \pm 0,0717^{\mathrm{NS}}$ & $0,0485 \pm 0,0185^{* *}$ & $0,1124 \pm 0,0626^{\mathrm{NS}}$ \\
\hline
\end{tabular}

$\mathrm{F}_{\mathrm{IT}}$ : desvios de panmixia para todas as amostras (população); $\mathrm{F}_{\mathrm{ST}}$ : desvios de panmixia devido à subdivisão do conjunto em subpopulações; $\mathrm{F}_{\mathrm{IS}}$ : desvios de panmixia dentro de subpopulações; NS = não significativo; $* *=\mathrm{P}<0,01$; Lap $=$ leucina aminopeptidase; Pep $1=$ peptidase 1 ; Pep2 $=$ peptidase 2; 6-Pgd = 6-fosfogluconato desidrogenase; $\mathrm{Mdh}=$ malato desidrogenase.

Apesar de várias subpopulações terem apresentado $\overline{\mathrm{f}}$ significativo $(\mathrm{P}<0,05)$, não foi significativo o índice médio de fixação intrapopulacional $\left(\mathrm{F}_{\mathrm{IS}}-\right.$ Tab. 3). Isso pode ser atribuído ao fato de essas populações estarem submetidas a diferentes sistemas de acasalamento.

O desvio médio da panmixia entre as subpopulações, medido pelo índice de fixação médio entre as subpopulações, $\mathrm{F}_{\mathrm{ST}}$, pode ser causado por dois fatores: endogamia dentro das subpopulações, determinando a formação de linhagens, e o efeito Wahlund. Uma vez que o valor desse índice, apesar de pequeno, foi significativo $(\mathrm{P}<0,05)$, pode-se supor que não esteja havendo suficiente troca de material genético entre as subpopulações.

Guiller et al. (1996) observaram, para amostras obtidas no Magreb, índice de fixação entre subpopulações superior ao encontrado neste trabalho $\left(\mathrm{F}_{\mathrm{ST}}=0,270 \pm 0,066\right)$, indicando subdivisão bem maior das amostras. Os índices médios de fixação intrapopulacional $\left(\mathrm{F}_{\mathrm{IS}}=\right.$ $0,170 \pm 0,038)$ e do conjunto de subpopulações $\left(\mathrm{F}_{\mathrm{IT}}=0,396 \pm 0,049\right)$ também foram maiores $\mathrm{e}$ significativos.

O número médio de alelos por loco polimórfico, o número efetivo de alelos por loco, a proporção de alelos contidos em cada população e a proporção de locos polimórficos, considerando polimórfico o loco cujo alelo mais freqüente não ultrapasse $\quad 99 \%$ das observações, são apresentados na Tab. 4. Os valores observados para esses quatro índices, em cada uma das amostras foram muito próximos, revelando uma baixa variabilidade genética nesse material.

Tabela 4. Número de alelos por loco polimórfico (A), número efetivo de alelos por loco $\left(\mathrm{A}_{\mathrm{e}}\right)$, proporção de alelos contidos em cada população (Pa) e proporção de locos polimórficos (P), considerando polimórfico o loco cujo alelo mais freqüente não ultrapassasse $99 \%$ das observações

\begin{tabular}{lcccc}
\hline Amostra & $\mathrm{A}$ & $\mathrm{A}_{\mathrm{e}}$ & $\mathrm{Pa}$ & $\mathrm{P}$ \\
\hline 1 & 2,6 & 1,7529 & 1,00 & 0,6250 \\
2 & 2,6 & 1,7750 & 1,00 & 0,6250 \\
3 & 2,6 & 1,6765 & 1,00 & 0,6250 \\
4 & 2,6 & 1,7846 & 1,00 & 0,6250 \\
5 & 2,4 & 1,6293 & 0,94 & 0,6250 \\
6 & 2,4 & 1,7147 & 0,94 & 0,6250 \\
7 & 2,4 & 1,7303 & 0,94 & 0,6250 \\
8 & 2,6 & 1,6793 & 1,00 & 0,6250 \\
9 & 2,6 & 1,7729 & 1,00 & 0,6250 \\
10 & 2,4 & 1,7937 & 0,94 & 0,5000 \\
11 & 2,6 & 1,7016 & 1,00 & 0,6250 \\
12 & 2,4 & 1,6378 & 0,94 & 0,5000 \\
13 & 2,6 & 1,8307 & 1,00 & 0,6250 \\
14 & 2,4 & 1,6683 & 0,94 & 0,6250 \\
15 & 2,6 & 1,8376 & 1,00 & 0,6250 \\
16 & 2,4 & 1,6490 & 0,94 & 0,6250 \\
17 & 2,4 & 1,7905 & 0,94 & 0,6250 \\
18 & 2,6 & 1,7637 & 1,00 & 0,6250 \\
19 & 2,4 & 1,8133 & 0,94 & 0,6250 \\
20 & 2,6 & 1,8121 & 1,00 & 0,6250 \\
\hline
\end{tabular}

As estimativas de diversidade genética (Tab. 5) indicam que a maior parte da diversidade observada encontra-se dentro das subpopulações. A diversidade média entre subpopulações $\left(\mathrm{D}_{\mathrm{ST}}=\right.$ 0,040118) foi muito pequena, à semelhança do que já havia sido indicado pelo $F_{\mathrm{ST}}$. Esse pequeno valor pode apontar que os criadores estão obtendo suas matrizes de poucos produtores ou que a base genética desse material é estreita. Essa observação apóia-se nos valores da Tab. 1, onde os números máximos de alelos observados por loco foram inferiores àqueles observados em estudos com populações nativas (Crook, 1982; Madec, 1991 a; Guiller et al, 1994; Arnaud et al. 1999).

Em geral, as distâncias genéticas entre as subpopulações foram muito pequenas, variando de 0,0024 a 0,093. Esses valores confirmam a pequena variabilidade genética entre populações. Valores muito superiores a estes, variando de 0,057 a 0,340 foram relatados por Guiller et al. (1994), em um estudo com 64 populações naturais obtidas na Europa e no norte da África. 
Os valores obtidos também foram inferiores àqueles relatados por Madec (1991 a), em um estudo com amostras provenientes de 15 populações naturais de diferentes regiões da Europa, da Árgélia e das ilhas La Reunion, no Oceano Índico, que variaram de 0,0302 a 0,3798.

Tabela 5. Estimativas de diversidade genética para cada loco e estimativas médias para todos os locos

\begin{tabular}{lcccc}
\hline & $\mathrm{H}_{\mathrm{T}}$ & $\mathrm{H}_{\mathrm{S}}$ & $\mathrm{D}_{\mathrm{ST}}$ & $\mathrm{G}_{\mathrm{ST}}$ \\
\hline LAP & 0,487596 & 0,436380 & 0,051216 & 0,105037 \\
PEP1 & 0,452380 & 0,439865 & 0,012515 & 0,027665 \\
PEP2 & 0,596265 & 0,563840 & 0,032425 & 0,054380 \\
6-PGD & 0,622781 & 0,596920 & 0,025861 & 0,017302 \\
MDH & 0,165168 & 0,086595 & 0,078573 & 0,009984 \\
Médias & 0,464838 & 0,424720 & 0,040118 & 0,042874 \\
\hline $\mathrm{H}_{\mathrm{T}}=$ diversidade total; $\mathrm{Hs}=$ & diversidade & dentro das \\
subpopulações; $\mathrm{D}_{\mathrm{ST}}=$ diversidade entre subpopulações; $\mathrm{G}_{\mathrm{ST}}=$ \\
proporção da diversidade total atribuível à subdivisão. (Nei, \\
1973).
\end{tabular}

As distâncias observadas não apresentaram heterogeneidade bastante para que os dendrogramas produzidos pela média das distâncias entre todos os pares de itens que formam cada grupo (Unweighted pair-group method using an arithmetic average - UPGMA) tivessem níveis de consistência estatisticamente significativos para os diferentes nós.

O fato de outros autores (Madec, 1991 a; Guiller, et al., 1994), que trabalharam com marcadores semelhantes aos utilizados neste estudo, terem observado distâncias entre as amostras suficientemente heterogêneas para permitir a construção de dendrogramas reforça a hipótese de que essas subpopulações provêm de uma mesma origem, com baixa variabilidade genética.

O melhoramento genético de uma espécie é obtido basicamente por seleção ou por cruzamento, que são estratégias complementares. Caso se opte pelo método da seleção, na implantação de um programa de melhoramento genético das características de interesse econômico para a espécie junto aos heliários estudados, é possível que a baixa variabilidade resulte em menor ganho genético, uma vez que ele diretamente depende da variabilidade genética da população onde a seleção é feita.

\section{CONCLUSÕES}

Os índices médios de fixação intraloco indicam que algumas das subpopulações estão sob endogamia que pode ser involuntariamente causada pela falta de controle reprodutivo nas criações. Os pequenos valores de distâncias genéticas entre as subpopulações, aliados às reduzidas estimativas de diversidade genética, sugerem que o material compartilha a mesma base genética e que ela provavelmente é estreita. Isso deverá ser levado em conta ao se definir o método de melhoramento a ser utilizado em cada subpopulação ou nas populações em conjunto.

\section{REFERÊNCIAS BIBLIOGRÁFICAS}

ALFENAS, A.C.; PETERS, I.; BRUNE, W. et al. Eletroforese de proteínas e isoenzimas de fungos e essências florestais. Viçosa: Universidade Federal de Viçosa, 1991. 242p.

ARNAUD, J.F.; MADEC, L.; BELLIDO, A. et al. Microspatial genetic structure in the land snail Helix aspersa (Gastropoda: Helicidae). Heredity, v.83, p.110-119, 1999.

ARNAUD, J.F.; MADEC, L.; DAGUZAN, J. Spatial differentiation of allozyme frequencies in a subdivided population of the land snail Helix aspersa. J. Moll. Stud., v.65, p.267-271, 1999.

CROOK, S.J. Genetic studies of allozyme variation in leucine aminopeptidase in the land snail Helix aspersa (Muller). J. Moll. Stud., v.48, p.362-365, 1982.

DUPONT-NIVET, M.; MALLARD, J.; BONNET, J. C et al. Quantitative genetics of reproductive traits in the edible snail Helix aspersa Müller. J. Exp. Zool., v.281, p.220-227, 1998.

FERREIRA, M.E; GRATTAPAGLIA, D. Introdução ao uso de marcadores moleculares em análise genética. 3.ed. Brasília: EMBRAPA - CENARGEN, 1998, 220p.

GUILlER, A.; COUTELEC-VRETO, M.A.; MADEC. L. Genetic relationships among suspected contact zone populations of Helix aspersa (Gastropoda: Pulmonata) in Algeria. Heredity, v.77, p.113 -129, 1996.

GUILLER, A.; MADEC, L. Variabilité enzimatique dans quelques populations naturelles 
de Cepaea nemoralis et Cepaea hortensis en France. Compte Rendu Acad. Sci. Paris, v.313, p.299-306, 1991.

GUILLER, A.; MADEC, L.; DAGUZAN, J. Geographical patterns of genetic differentiation in the land snail Helix aspersa Müller (Gastropoda: Pulmonata). J. Moll. Stud., v.60, p.205-221, 1994.

GUO, S.W.; THOMPSON, E.A. Performing the exact test of Hardy-Weinberg proportion for multiple alleles. Biometrics, v.48, p.361-372, 1992.

HARRIS, H.; HOPKINSON, D. Handbook of enzyme electrophoresis in human genetics. Amsterdam: North-Holland Publishing, 1977. $567 \mathrm{p}$.

LAZARIDOU-DIMITRIADOU, M.; ALPOYANNI, E.; BAKA, M. et al. Growth, mortality and fecundity in successive generations of Helix aspersa Müller cultured indoors and crowding effects on fast, medium and slow-growing snails of the same clutch. J. Moll. Stud., v.64, p.67-74, 1998.

MADEC, L. Genetic divergence in natural populations of the landsnail Helix aspersa Müller, 1774. J. Moll. Stud., v.57, p.483-487. 1991a.
MADEC, L. Variabilité enzimatique dans quelques populations naturelles de l'espèce Helix aspersa Müller. Compte Rendu Acad. Sci. Paris, v.313, p.339-344, $1991 \mathrm{~b}$.

MILLER, M.P. Tools for population genetic analysis (TFPGA) 1.3: A Windows program for the analysis of allozyme and molecular population genetic data. Department of Biological Sciences, Northern Arizona University, Flagstaff, Arizona. Disponível em: $<$ http://www.public.asu.edu/ mmille8/>. Acessado em: 15 jul. 2001.

NEI, M. Analysis of gene diversity in subdivided populations. Proc. Nat. Acad. Sci. USA, v.70, p.3321-3323, 1973.

NEI, M. Genetic distance between populations. Am. Natur., v.106, p.283-292, 1972.

ROBINSON, I.P. Aloenzimas na genética de populações de plantas. In: ALFENAS, A.C. (Ed.). Eletroforese de isoenzimas e proteinas afins. Universidade Federal de Viçosa, 1998. p.328-367.

WEIR, B.S.; COCKERHAM, C.C. Estimating Fstatistics for the analysis of population structure. Evolution, v.38, p.1358-1370, 1984. 